\title{
Primeira ocorrência de Drosera cayennensis Sagot ex Diels (Droseraceae) nas campinas do baixo rio Tocantins, estado do Pará, como subsídio à criação de novas unidades de conservação \\ First occurrence of Drosera cayennensis Sagot ex Diels (Droseraceae) in the white sand campinas of the lower Tocantins River, state of Pará, such support for the creation of new protected areas
}

\author{
Leandro Valle Ferreira!', João Ubiratan Moreira dos Santos", \\ Maria de Nazaré do Carmo Bastos', Denise de Andrade Cunha' \\ IMuseu Paraense Emílio Goeldi/MCTI. Belém, Pará, Brasil \\ "Universidade Federal Rural da Amazônia/MEC. Belém, Pará, Brasil
}

\begin{abstract}
Resumo: As campinas amazônicas são um dos tipos de vegetação mais ameaçados e menos protegidos no estado do Pará, devido à perda de habitats pela extração de areia. Essa vegetação ocupa menos de 2\% do Pará, sendo caracterizada pelo pequeno porte da vegetação sob solos arenosos, extremamente pobres em nutrientes, com elevado número de espécies endêmicas e indicadoras da qualidade ambiental. Entre essas, destaca-se Drosera cayennensis Sagot ex Diels, uma planta carnívora. Em um levantamento no Herbário do Museu Paraense Emílio Goeldi (MG), foram encontrados somente sete registros dessa espécie no estado do Pará, a maioria no município de Vigia, onde atualmente as campinas estão em péssimo estado de conservação. Nesse estudo, é relatada a primeira ocorrência de $D$. cayennensis em uma campina em bom estado de conservação no município de Cametá. Atualmente, essas campinas estão sendo destruídas para a extração de areia, processo semelhante ao ocorrido nas campinas do município de Vigia. A descoberta de D. cayennensis nas campinas do municíio de Cametá demonstra a qualidade ambiental da área, sua importância para a conservação da flora e serve como subsídio para a criação de uma nova unidade de conservação, antes que essas campinas sejam destruídas.
\end{abstract}

Palavras-chave: Campina. Drosera. Perda de habitats. Unidade de conservação.

\begin{abstract}
The White-sand vegetation (campinas) of Amazonia are one of the most threatened and least protected vegetation types in the State of Pará by extraction of sand used in construction. White-sand vegetation occupies less than 2\% of the Pará. The sandy soils poor in nutrients and harbour a high number of endemic species which can act as indicators of environmental quality. Among these species is Drosera cayennensis Sagot ex Diels (Droseraceae), a carnivorous plant. In a survey held in the Herbarium of the Museu Paraense Emílio Goeldi, only seven records were found for Pará, most of which were located in the campinas municipality of the Vigia, that is currently in a poor state of preservation. The first occurrence of $D$. cayennensis in a campinas in good condition is reported in the municipality of Cametá in Pará. However, at present these campinas are being destroyed from sand extraction, a similar process that resulted in the destruction of the campinas in the municipality of Vigia. The discovery of $D$. cayennensis in campinas of the municipality of Cametá demonstrates the conservation importance of the area, and provides further justification for the creation of a new unit of conservation before the area is irreversibly impacted.
\end{abstract}

Keywords: White-sand vegetation. Drosera. Habitat loss. Conservation unit.

FERREIRA, L. V., J. U. SANTOS, M. N. C. BASTOS \& D. A. CUNHA, 2013. Primeira ocorrência de Drosera cayennensis Sagot ex Diels (Droseraceae) nas campinas do baixo rio Tocantins, estado do Pará, como subsídio à criação de novas unidades de conservação. Boletim do Museu Paraense Emílio Goeldi. Ciências Naturais 8(2): 223-230.

Autor para correspondência: Leandro Valle Ferreira. Museu Paraense Emílio Goeldi/MCTI. Coordenação de Botânica. Avenida Perimetral, 1901 - Terra Firme. CEP 66077-530. Belém, PA, Brasil (lvferreira@museu-goeldi.br).

Recebido em 11/05/2012

Aprovado em 18/06/2013

Responsabilidade editorial: Toby Gardner

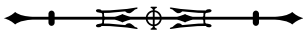




\section{INTRODUÇÃO}

As formações abertas da Amazônia (savanas, campos rupestres, campinas, restingas costeiras) são fisionomias tropicais nas quais uma camada herbácea é dominante e onde arbustos e árvores podem ou não estar presentes, sendo representadas, na Amazônia, em grande parte, por encraves isolados de diferentes tamanhos (Veloso et al., 1991).

A vegetação de campina amazônica ocorre em solos arenosos, denominados podzóis hidromórficos (Anderson, 1981). É caracterizada pelo pequeno porte, distribuída em moitas de diferentes tamanhos, em cujo solo são encontradas diversas espécies de bromélias, orquídeas e liquens (Anderson, 1981), além de algumas espécies insetívoras, plantas características de solos pobres em nutrientes (Araújo et al., 2007).

No Pará, os fragmentos de vegetação de campina estão distribuídos irregularmente, formando manchas de diferentes dimensões e graus de isolamento, ocupando menos de $2 \%$ do estado, a maioria delas fora do atual sistema de áreas protegidas (Ferreira et al., 2010).

Estudos prévios realizados na vegetação de campina na Amazônia relatam que ela possui uma flora regional especializada, cujas espécies apresentam padrões de distribuição geográficos bem delimitados, com comunidades animais e vegetais únicas, apresentando, ainda, muitas espécies restritas a determinadas áreas (Ferreira, 2009).

No estado do Pará, Hopkins (1986) registrou nas campinas da Serra do Cachimbo algumas espécies de plantas endêmicas, tais como Parkia cachimboensis H.C. Hopkins (Mimosaceae) e Blepharandra cachimbensis W.R. Anderson (Malpighiaceae), demonstrando a grande importância desses tipos de vegetação na conservação da flora amazônica.

O gênero Drosera tem cerca de 150 espécies, das quais 16 foram registradas no Brasil. São plantas adaptadas a diversos tipos de habitats (Juniper et al., 1989) e exigentes quanto à qualidade ambiental deles, sendo frequentemente usadas como espécies indicadoras dessa qualidade.
Entre essas espécies, Drosera cayennensis Sagot ex Diels (Droseraceae) é característica da vegetação de campinas amazônicas, enquanto Drosera capillaris Poir. e D. tenella Willd. ex Roem. \& Schult, das restingas litorâneas amazônicas no estado do Pará (Amaral et al., 2008).

As campinas amazônicas estão atualmente entre os tipos de vegetação mais ameaçados pela perda de habitats (Ferreira et al., 2010). A fragmentação de habitats, inclusive, é uma das mais importantes e difundidas consequências da atual dinâmica de uso da terra produzida pelo homem, sendo considerada uma das principais causas de perda da biodiversidade na região (Laurance, 2001).

As campinas e outros tipos de vegetações abertas no estado do Pará, tais como savanas, campos rupestres e restingas costeiras, estão entre as fisionomias com menor representatividade de área incluídas no atual sistema de unidades de conservação neste estado (Ferreira et al., 2010).

Isso tem implicações importantes para a conservação. Ferreira et al. (2005) demonstraram que o desmatamento dentro das unidades de conservação e de terras indígenas na Amazônia é sempre menor do que fora delas, relação essa variando de dez, no estado de Mato Grosso, a 20 vezes, no estado do Pará.

Ferreira et al. (2010) recomendaram que a vegetação de campinas da região do baixo Tocantins e do município paraense de Acará deva ser protegida com a criação de novas unidades de conservação, pois ela tem extensão extremamente reduzida, sendo uma comunidade biótica ecologicamente única, em função das adaptações às condições ambientais, e representando um dos ecossistemas amazônicos mais frágeis e vulneráveis a atividades humanas.

No caso das campinas amazônicas, é urgente a criação de novas unidades de conservação para sua proteção. Tabarelli \& Gascon (2005) relatam que a priorização de áreas a serem transformadas em unidades de conservação deveria ser feita a partir de critérios de importância biológica, necessitando ocorrer antes que grandes extensões de habitats sejam modificadas definitivamente pela ação humana ou tenham desaparecido 
(Margules \& Pressey, 2000), tal como ocorreu nas campinas do município de Vigia, na Zona Bragantina do Pará (Vieira et al., 1967), e está acontecendo com as campinas do município de Acará, no nordeste paraense.

Seguindo essas recomendações, a Secretaria de Estado do Meio Ambiente do Pará está avaliando propostas de criação de unidades de conservação nas campinas do baixo rio Tocantins.

A descoberta de Drosera cayennensis Sagot ex Diels (Droseraceae) nas campinas do baixo rio Tocantins, no estado do Pará, reforça a importância da conservação dessas formações vegetais, que, atualmente, têm sua preservação altamente comprometida pela degradação ambiental causada pela crescente atividade de extração de areia usada na construção civil e têm pouca representatividade em termos de área protegida em unidades de conservação.

\section{MATERIAL E MÉTODOS}

Os espécimes de $D$. cayennensis foram identificados na vegetação de campinas à margem direita do rio Tocantins (208' 27” S; $49017^{\prime}$ 44” W), no município paraense de Cametá, na rodovia PA-467, a cerca de $15 \mathrm{~km}$ da vila de Curuçambaba (Figura 1).

Pela classificação de Köppen, o clima do município corresponde ao tipo Ami, com média mensal da temperatura mínima superior a $18{ }^{\circ} \mathrm{C}$ e amplitude térmica que não ultrapassa $5^{\circ} \mathrm{C}$. A umidade relativa está sempre acima de $80 \%$. A precipitação pluviométrica é de $2.200 \mathrm{~mm}$ anuais, sendo o mês de abril considerado o de maior pluviosidade, com registro de até $441 \mathrm{~mm}$, e o mês de novembro o menos chuvoso, quando já foram registrados apenas $9 \mathrm{~mm}$ (IDESP, 2013).

O levantamento dos espécimes de Drosera cayennensis Sagot ex Diels (Droseraceae) foi realizado no Herbário do Museu Paraense Emílio Goeldi (MG). Fundado em 1895 pelo botânico suíço Jacques Huber, o Herbário MG é a principal coleção da Coordenação de Botânica do Museu Paraense Emílio Goeldi, sendo também o primeiro da região amazônica e o terceiro mais antigo do Brasil (Cavalcante, 1984).
Os espécimes de Drosera cayennensis foram encontrados em uma vegetação de campina, caracterizada pela presença de poucas e espaçadas árvores, solos arenosos pobres em nutrientes, sujeitos a inundação durante o período chuvoso e com grande quantidade de plantas herbáceas, formando um tapete contínuo (Figura 2), representadas principalmente pelas espécies Syngonanthus tenuis var. bulbifer (Huber), Paepalanthus fertilis Körn. (Eriocaulaceae), Rhynchospora barbata (Vahl) Kunth e Lagenocarpus rigidus Nees (Cyperaceae) (Ferreira et al., 2010).

Essa vegetação é denominada localmente de "campos gerais", formações herbáceas graminosas a subarbustivas que se desenvolvem em solos arenosos de natureza hidromórfica. Ressalta-se que a utilização de flores decorativas ocorrentes nesses campos vem se constituindo em importante atividade econômica para o estado do Pará, inclusive no setor de exportação (IDESP, 2013).

\section{RESULTADOS E DISCUSSÃO}

No Herbário do Museu Paraense Emílio Goeldi (MG), foram encontrados quinze registros de $D$. cayennensis, dos quais sete foram coletados no estado do Pará, todos em áreas de vegetação de campina sujeitas à inundação: cinco no município de Vigia, na região nordeste do estado; um registro no município de Alenquer, na calha norte do estado; e um registro no município de Altamira, a sudoeste do estado, próximo ao estado do Mato Grosso (Tabela 1).

Os espécimes coletados no presente estudo compõem o oitavo registro no Herbário MG e o primeiro de $D$. cayennensis na vegetação de campina à margem direita da região do baixo rio Tocantins, no estado do Pará, que se encontra em bom estado de conservação.

Os exemplares foram coletados na estação climática de seca, em outubro de 2009, sob uma camada orgânica, formada principalmente pela decomposição de partes vegetativas de plantas, onde ainda havia vestígios de umidade (Figuras 3A e 3B).

A descoberta de $D$. cayennensis em campinas do baixo Tocantins tem grande importância para a 




Figura 1. Localização da campina da região do baixo rio Tocantins, próximo à Vila de Curuçambaba, no estado do Pará. Mapa: Leandro V. Ferreira, 2012.

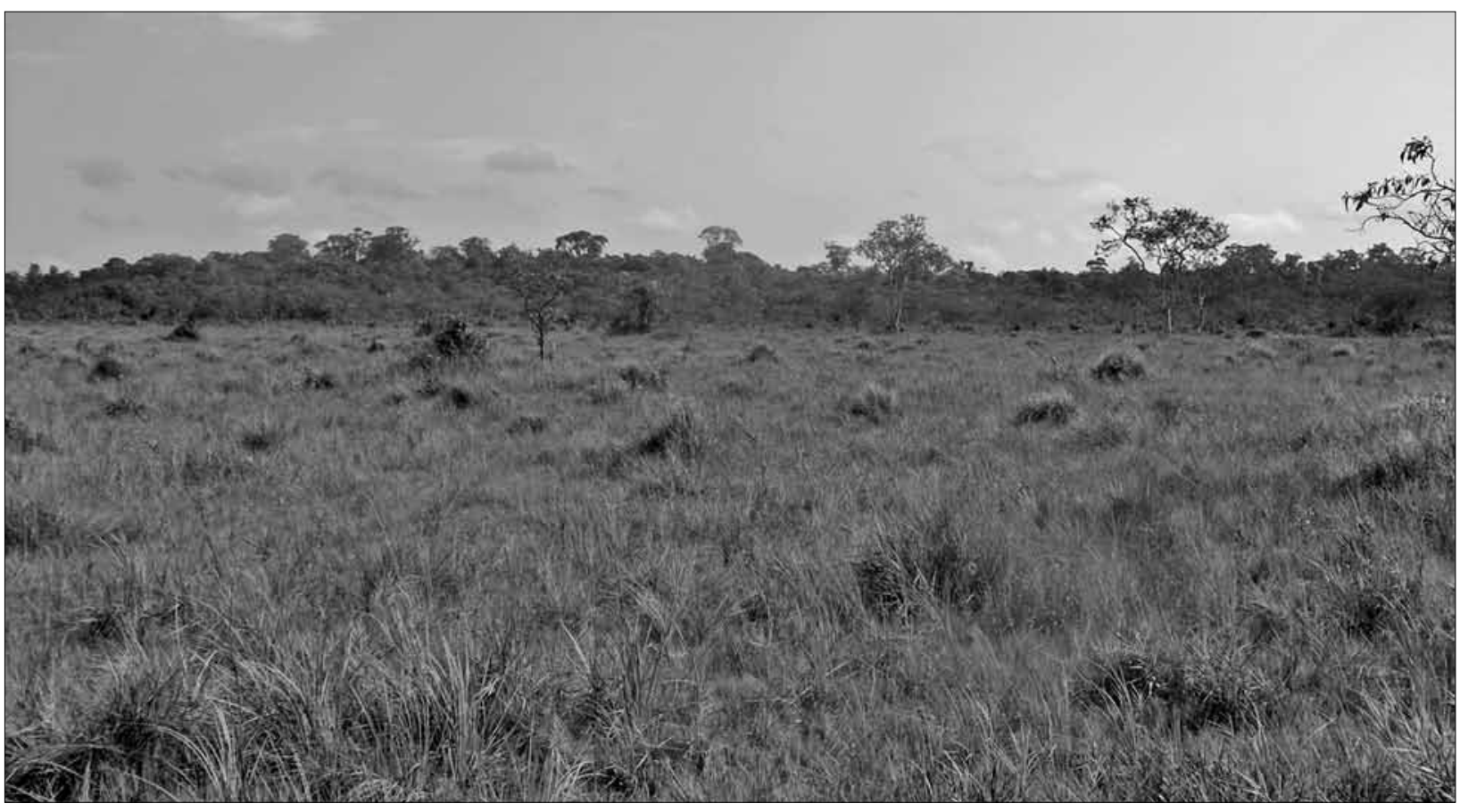

Figura 2. Estrutura da vegetação de campina na região do baixo rio Tocantins, no município de Cametá, Pará. Foto: Leandro V. Ferreira, 2009.

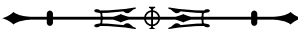


Tabela 1. Localização das coletas de Drosera cayennensis Sagot ex Diels (Droseraceae) no estado do Pará depositadas no Herbário do Museu Paraense Emílio Goeldi (MG).

\begin{tabular}{c|c|c|c}
\hline Registro do Herbário & Município & Local de coleta & Tipo de vegetação \\
\hline 22402 & Alenquer & Taboleta; campos do Ariramba & Campina \\
\hline 22701 & Vigia & Estrada da Vigia, Campina do Palha & Campina \\
\hline 23891 & Vigia & Vigia, Campo do Palha & Campina \\
\hline 37982 & Vigia & Estrada da Vigia, km 80 & Campina \\
\hline 46108 & Vigia & Vigia, estrada da Vigia & Campina \\
\hline 45091 & Vigia & Santa Izabel, estrada Santa Izabel-Vigia, km 30 & Campina \\
\hline 59880 & Altamira & BR-163, km 764, 15 km ao sul de & Mato Grosso, fronteira com o Pará \\
\hline
\end{tabular}

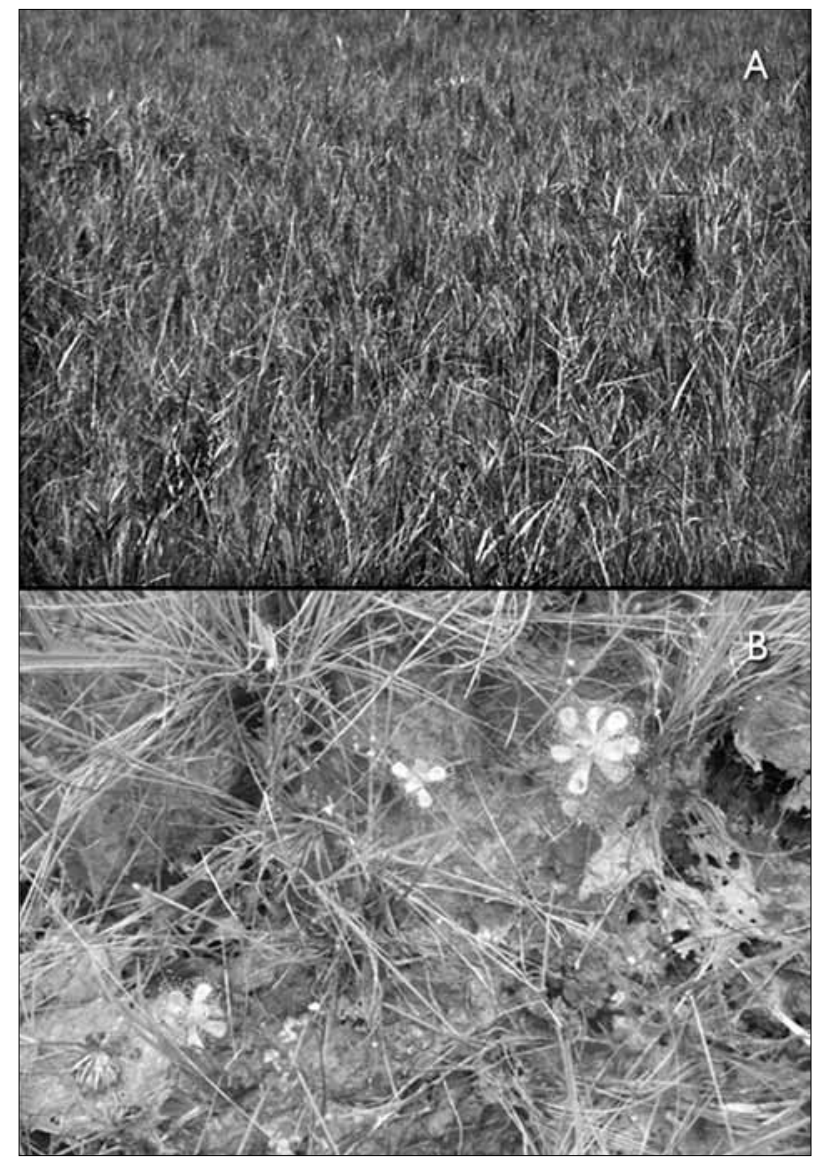

Figura 3. Foto mostrando a camada herbácea com domínio de Lagenocarpus rigidus Nees (Cyperaceae) nas campinas do baixo rio Tocantins, município de Cametá, Pará (A); habitat preferencial de Drosera cayennensis Sagot Ex Diels (B). conservação dessa e de outras espécies da flora, além da fauna associada a esse tipo de vegetação, pois, atualmente, essas campinas estão sendo impactadas pela ação humana, principalmente ligada à exploração de areia para a construção civil e obras de infraestrutura (Figuras 4A e 4B).

O mesmo processo ocorreu na Zona Bragantina do Pará, nas campinas do município de Vigia, que atualmente se encontra em elevado grau de degradação. Vieira et al. (1967) relatam que a vegetação de campina nessa região foi destruída pela exploração da areia. Segundo Ferreira et al. (no prelo), a mesma perda de habitats está ocorrendo nas campinas do município de Acará, no nordeste paraense, onde a extração de areia está abastecendo a construção civil no município de Belém.

Apesar de nenhuma espécie do gênero Drosera constar na lista de espécies da flora brasileira ameaçada de extinção (IBAMA, 2012), Drosera graomogolensis T. R. S. Silva, encontrada nos campos rupestres em Minas Gerais, consta no grupo de espécies cujas informações (distribuição geográfica, ameaças/impactos e usos, entre outras) são ainda deficientes, o que não permite enquadrá-las com segurança na condição de espécies ameaçadas de extinção.






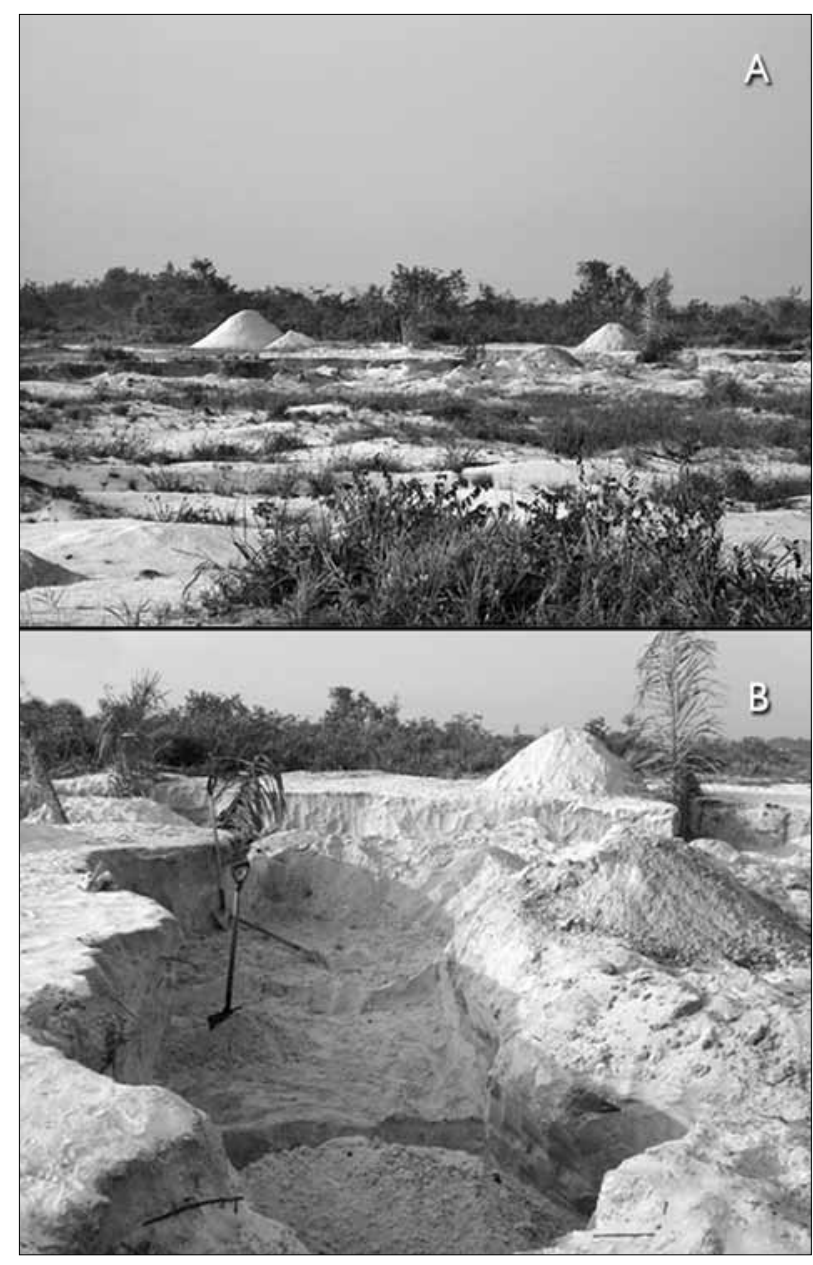

Figura 4. Foto mostrando o resultado do impacto de extração de areia na vegetação de campina na região do baixo rio Tocantins no município de Cametá $(A)$ e detalhe da escavação, mostrando o solo composto por areia branca (B). Fotos: Leandro V. Ferreira, 2009.

Nesse estudo, sugere-se que o mesmo critério seja aplicado à $D$. cayennensis, pois a distribuição geográfica dessa espécie ainda é pouco conhecida e os habitats onde ocorre estão sendo impactados por atividades humanas, incorporando as ideias de Ellison \& Gotelli (2001), os quais sugerem que as pesquisas sobre plantas carnívoras podem orientar a conservação e as estratégias de gestão para essas plantas, cada vez mais ameaçadas por coletas, destruição de habitats e mudanças ambientais.
Nesse estudo, recomenda-se a criação de uma unidade de conservação na região onde foram encontrados os espécimes de $D$. cayennensis. Esta unidade de conservação seria enquadrada no grupo de manejo de uso sustentável, em que a exploração dos recursos naturais da área, por exemplo, a extração de areia, possa ser feita de maneira a garantir a perenidade dos recursos ambientais e manter os processos ecológicos, fundamentais para a conservação da biodiversidade em longo prazo.

A categoria sugerida para a criação é uma Área de Relevante Interesse Ecológico (ARIE), definida como uma região de pequena extensão, com pouca ocupação humana, podendo ser constituída por terras públicas ou privadas, como já relatado por Ferreira et al. (2010) no Zoneamento Ecológico-Econômico do estado do Pará (ZEE-PA).

Nessa região, o ZEE-PA sugeriu cinco áreas para a criação de novas unidades de conservação, com dimensões variando de 8,4 mil a 64,8 mil hectares (Figura 5), adequadas para a conservação da biota desse tipo de vegetação.

A criação dessas unidades de conservação vai permitir a preservação de parte das campinas da região do baixo rio Tocantins, onde não existem unidades de conservação protegendo esse tipo de vegetação. As únicas áreas protegidas existentes são a Reserva Extrativista Arióca-Pruanã e a Terra Indígena Anambé, com, respectivamente, 59,6 mil e 21,7 mil hectares, as quais preservam outros tipos de vegetação.

Essas unidades de conservação, se criadas, poderão preencher uma lacuna importante na proteção da biota dessa região, pois na campina amazônica desenvolve-se uma vegetação com características naturais extraordinárias, abrigando exemplares raros, cuja preservação vai contribuir na manutenção dos ecossistemas naturais de importância regional ou local e regulamentar o uso econômico dessas áreas, de modo a compatibilizá-lo com os objetivos de conservação.

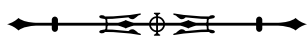




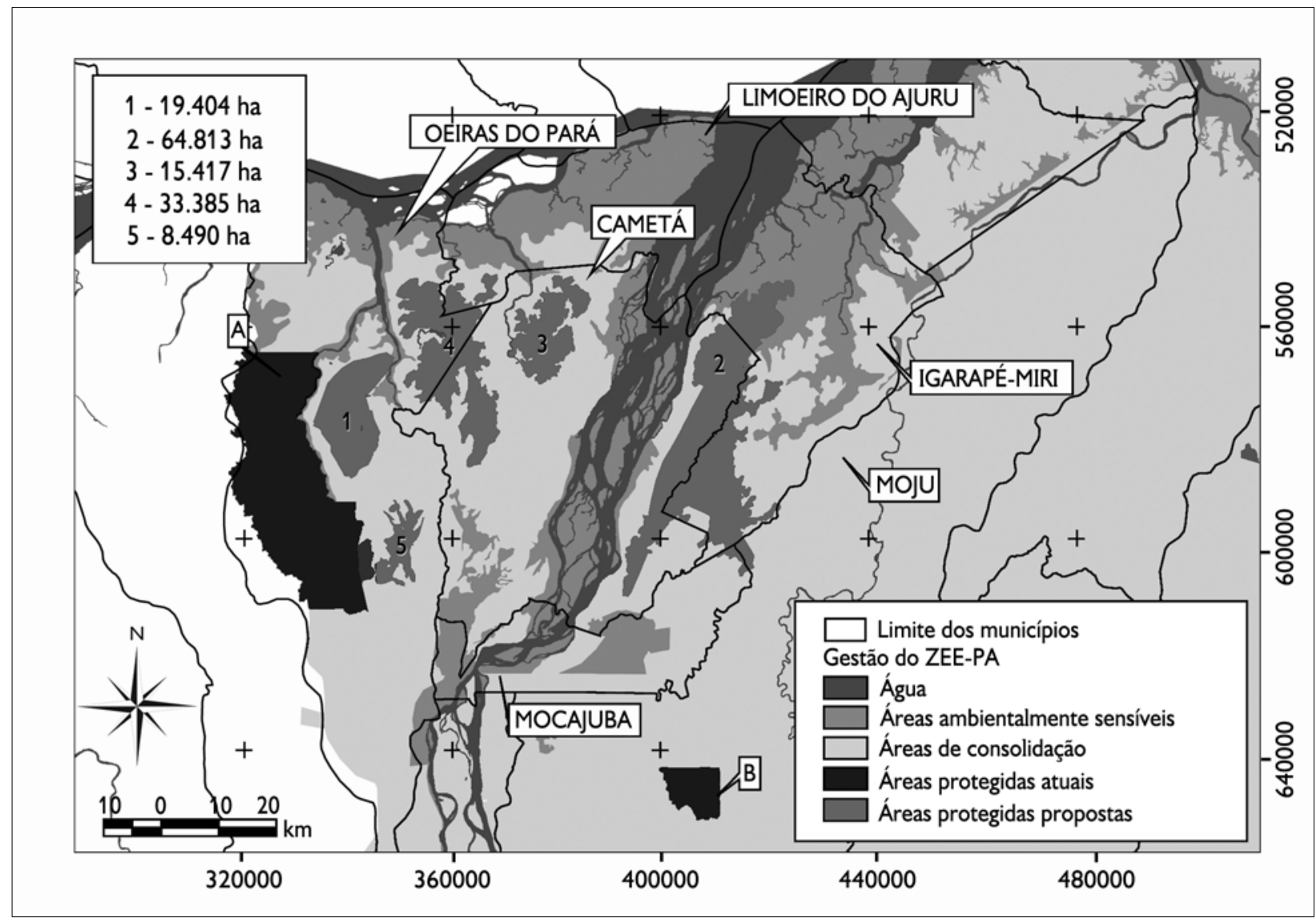

Figura 5. Mapa do Zoneamento Ecológico-Econômico do estado do Pará, mostrando a localização das novas áreas protegidas propostas neste estudo, que contemplam as campinas e savanas na região do baixo rio Tocantins, em uma região que tem apenas duas áreas protegidas, a Reserva Extrativista Arióca-Pruanã e a Terra Indígena Anambé, com 59,6 mil e 21,7 mil hectares, respectivamente. Mapa: Leandro V. Ferreira, 2012.

\section{CONCLUSÃO}

A descoberta da espécie Drosera cayennensis Sagot ex Diels (Droseraceae) nas campinas da região do baixo rio Tocantins indica que a área é importante para a conservação por dois motivos: (1) a vegetação de campina que se desenvolve na região ainda apresenta grande integridade ambiental, apesar do grau de ameaça atual; (2) tal descoberta amplia a distribuição da Drosera cayennensis Sagot ex Diels (Droseraceae) para uma região ainda não protegida no atual sistema de unidades de conservação do estado do Pará.

A criação de novas unidades de conservação na região do baixo rio Tocantins será fundamental para a preservação das campinas, pois elas têm extensões reduzidas, comunidades bióticas ecologicamente únicas, em função das adaptações às condições ambientais, e representam um dos ecossistemas amazônicos mais frágeis e vulneráveis às atividades humanas.

\section{AGRADECIMENTOS}

Aos funcionários do Museu Paraense Emílio Goeldi, L. C. B. Lobato e L. S. Silva, pelo auxílio na coleta de dados, e à Secretaria de Estado de Projetos Estratégicos (SEPE) do estado do Pará, pelo Convênio de Cooperação Técnica n 003/2008 entre o Núcleo de Gerenciamento do Programa Pará Rural e o Museu Paraense Emílio Goeldi, que financiou as expedições desse estudo.

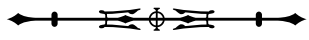




\section{REFERÊNCIAS}

AMARAL, D. D., M. T. PROST, M. N. C. BASTOS, S. V. C. NETO \& J. U. M. SANTOS, 2008. Restingas do litoral amazônico, estados do Pará e Amapá, Brasil. Boletim do Museu Paraense Emílio Goeldi. Ciências Naturais 3(1): 35-67.

ANDERSON, A. B., 1981. White-sand vegetation of Brazilian Amazonian. Biotropica 13(3): 199-210.

ARAÚJO, R. S., P. H. D. LEMOS, T. S. COSER, J. A. NUNES, M. N. DELGADO, M. A. MONTE, E. P. GUSMÃO, J. S. ARAÚJO, I. M. C. RODRIGUES, E. A. GUAÇONE \& J. A. A. M. NETO, 2007. Plantas carnívoras ocorrentes na Cachoeira Sempre-Viva do Parque Estadual do Rio Preto (PERP), MG. Revista Brasileira de Biociências 5(S2): 687-689.

CAVALCANTE, P. B., 1984. O Herbário do Museu Goeldi: 1-26. CNPQ/MPEG (Série Guias, 6), Belém.

ELLISON, A. \&J. GOTELLI, 2001. Evolutionary ecology of carnivorous plants. Trends in Ecology and Evolution 11(16): 623-629.

FERREIRA, C. A. C., 2009. Análise comparativa de vegetação lenhosa do ecossistema campina na Amazônia brasileira: 1-277. Tese (Doutorado em Botânica) - Instituto Nacional de Pesquisas da Amazônia (INPA), Manaus. Disponível em: <www.dominiopublico. gov.br/pesquisa/DetalheObraForm.do? select_action = \&co obra $=158624>$. Acesso em: 25 setembro 2012.

FERREIRA, L. V., E. VENTICINQUE \& S. S. ALMEIDA, 2005. O desmatamento na Amazônia e a importância das áreas protegidas. Estudos Avançados 19(53): 157-166.

FERreirA, L. V., M. C. THALES, J. L. G. PEREIRA, J. A. FERNANDES, C. FURTADO \& P. P. CHAVES, 2010. Biodiversidade. In: M. A. MONTEIRO, C. R. C. MENEZES \& I. M. F. GALVÃO (Orgs.): Zoneamento Ecológico-Econômico da Zona Leste e Calha Norte do Estado do Pará: diagnóstico do meio físico-biótico: 25-102. Secretaria de Estado de Projetos Estratégicos/Núcleo de Gerenciamento do Programa Pará Rural, Belém.

FERREIRA, L. V., P. P. CHAVES, A. A. CUNHA, A. S. ROSÁRIO \& P. PAROLIN, no prelo. A extração ilegal de areia como causa do desaparecimento de campinas e campinaranas no Estado do Pará, Brasil. Pesquisas, série Botânica.
HOPKINS, H. C. F., 1986. Parkia (Leguminosae: Mimosoideae). Flora Neotropica. Monograph 43: 1-124.

INSTITUTO BRASILEIRO DO MEIO AMBIENTE E DOS RECURSOS NATURAIS RENOVÁVEIS (IBAMA), 2012. Instrução Normativa $n^{\circ}$ 06, de 23 de setembro de 2008. Disponível em: <http://www.ibama.gov.br/index.php?option=com phocadownload\&view = category\&download =999: $06-$ 2008.p\&id=47: \&ltemid=331>. Acesso em: 25 setembro $\overline{2} 012$.

INSTITUTO DE DESENVOLVIMENTO ECONÔMICO, SOCIAL E AMBIENTAL DO PARÁ (IDESP), 2013. Estatística municipal Cametá: 6-44. Disponível em: <www.idesp.pa.gov.br/paginas/ produtos/EstatisticaMunicipal/pdf/Cameta.pdf>. Acesso em: 25 setembro 2012.

JUNIPER, B. E., R. J. ROBINS \& D. M. JOEL, 1989. The carnivorous plants. Academic Press, London.

LAURANCE, W. F., 2001. Fragmentation and plant communities: synthesis and implications for landscape management. In: R. O. BIERREGAARD JR., C. GASCON, T. E. LOVEJOY \& R. MESQUITA (Eds.): Lessons from Amazonian: the ecology and conservation of a fragmented forest: 158-168. Yale University Press, New Haven.

MARGULES, C. R. \& R. L. PRESSEY, 2000. Systematic conservation planning. Nature 405: 243-253.

TABARELLI, M. \& C. GASCON, 2005. Lições da pesquisa sobre fragmentação: aperfeiçoando políticas e diretrizes de manejo para a conservação da biodiversidade. Megadiversidade 1(1): 181-188.

VELOSO, H. P., A. L. R. RANGEL FILHO \& J. C. A. LIMA, 1991. Classificação da vegetação brasileira, adaptada a um sistema universal: 1-124. IBGE, Rio de Janeiro.

VIEIRA, L. S., W. H. F. SANTOS, I. C. FALESI \& J. P. S. OLIVEIRAFILHO, 1967. Levantamento de reconhecimento dos solos da região bragantina, estado do Pará: 1-68. Instituto de Pesquisas e Experimentação Agropecuária do Norte (Boletim Técnico, 47), Belém.

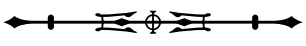

